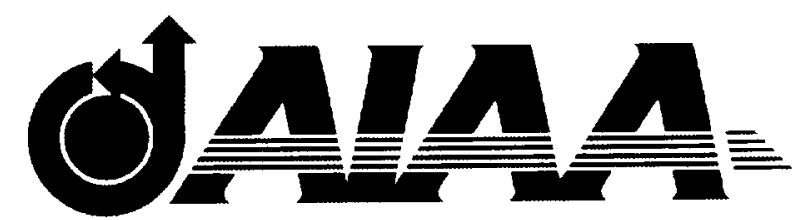

\section{AIAA 2000-2031}

\section{Noise Prediction For Maneuvering Rotorcraft}

Kenneth S. Brentner

NASA Langley Research Center

Hampton, Virginia

Henry E. Jones

U.S. Army Aeroflightdynamics Directorate

AMRDEC, U. S. Army Aviation and Missile Command NASA Langley Research Center Hampton, Virginia

\section{6th AIAA/CEAS Aeroacoustics Conference 12-14 June 2000 / Lahaina, Hawaii}




\title{
NOISE PREDICTION FOR MANEUVERING ROTORCRAFT
}

\author{
Kenneth S. Brentner ${ }^{*}$ \\ NASA Langley Research Center \\ Hampton, Virginia \\ Henry E. Jones ${ }^{\dagger}$ \\ U.S. Army Aeroflightdynamics Directorate \\ AMRDEC, U. S. Army Aviation and Missile Command \\ NASA Langley Research Center \\ Hampton, Virginia
}

\begin{abstract}
This paper presents the initial work toward firstprinciples noise prediction for maneuvering rotors. Both the aeromechanical and acoustics aspects of the maneuver noise problem are discussed. The comprehensive analysis code, CAMRAD 2, was utilized to predict the time-dependent aircraft position and attitude, along with the rotor blade airloads and motion. The major focus of this effort was the enhancement of the acoustic code WOPWOP necessary to compute the noise from a maneuvering rotorcraft. Full aircraft motion, including arbitrary transient motion, is modeled together with arhitrary rotor blade motions. Noise from a rotorcraft in turning and descending flight is compared to level flight. A substantial increase in the rotor noise is found both for turning flight and during a transient maneuver. Additional enhancements to take advantage of parallel computers and clusters of workstations, in addition to a new compact-chordwise loading formulation. are also described.
\end{abstract}

\section{INTRODUCTION}

The ability to predict rotorcraft noise has advanced greatly in the past two decades. In particular, deterministic noise sources are well understood and the accurate prediction of non-impulsive noise (i.e.. thickness and loading noise) can be accomplished routinely and with great confidence-for steady rectilinear flight. The prediction of impulsive noise. such as blade-vortex-interaction (BVI) noise and high-

\footnotetext{
${ }^{*}$ Senior Research Engineer. Computational Modeling and Simulation Branch, Senior Member AIAA.

Research Scientist. Subsonic Aerodynamic Branch. Senior Member AIAA.

Copyright $\bigcirc 2000$ hy the American Institute of Acronautics and Astronautics. Inc. No copyright is asserted in the United States under Title 17. U.S. Code. The U. S. Government has a royalty-free license to exercise all rights under the copyright herein for Governmental Purposes. All other rights are reserved by the copyright owner.
}

speed-impulsive (HSI) noise, is more difficult, hut only because the prediction of the rotor flow field and dynamical state is still challenging.

The problem of predicting noise for a rotorcraft in a maneuver, however, still poses a daunting and largely untackled challenge. In both civil and military operations. maneuvering flight is essential for a rotorcraft to perform its intended mission. Nevertheless, current rotor-noise-prediction methods do not fully address even the simplest maneuvers. such as accelerating and decelerating flight in rectilinear motion. The real-world situation, however, is considerably more complex, involving unsteady. nonperiodic conditions and the transient effects associated with control inputs and short-period maneuvers (e.g. a pull-up). Unsteady aircraft motions-such as pitch. roll, or yaw motions-cause significant time-dependent shifts in the noise directivity, much like a flashlight sweeping across a scene during a nightlime search. Furthermore, transient maneuvers can generate a significant increase in the noise radiation due to both kinematic and aerodynamic effects.

Recently Gopalan et al. ${ }^{1}$ has developed a method to predict the first-order effects of acceleration (deceleration) parallel to the flight path. JanakiRam and $\mathrm{Khan}^{2}$ have performed a detailed state-of-the-art prediction and validation for a helicopter in descending and decelerating flight. In both of these works, quasisteady analyses (i.e., predictions for a series of steady trim states made at several points along the flight path) were used for flight conditions where the aircraft state varied along a straight flight path. Aircraft acceleration was included in the trim computation, but it was not represented in the noise prediction directly.

This paper addresses some of the unique aspects. and difficulties of predicting the rotor noise of a maneuvering rotoreraft, and the significant impact maneuver has on rotor noise. The primary goal of this paper is to outline the development of a new version of the rotor-noise-prediction code WOPWOP ${ }^{3}$ that is capable of computing the noise due to a transient 
maneuver. The maneuver-noise-prediction capability will be demonstrated for several simple maneuvers, including both steady and transient flight conditions. A secondary goal of the paper is to explore the fitness of state-of-the-art rotorcraft comprehensive analysis codes for maneuvering flight. It will be impossible in this paper to even identify all of the physical and numerical modeling issues associated with maneuvering flight, yet it is hoped that this work will demonstrate the importance of further research in this area.

\section{ManeUVer NoISE PREDICTION METHODOLOGY}

Maneuvering flight conditions can be divided into two general categories: 1) steady maneuvers (e.g. a coordinated turn), and 2) transient maneuvers (e.g. transition to a turn or a pull-up). The steady maneuver is similar to steady flight, but with the additional complexity of acceleration (or deceleration) of the rotorcraft duc to either a curved flight path or a constant change in aircraft velocity. In particular, this type of flight condition exhibits the same periodic behavior as normal level flight-hence most of the modeling assumptions used in current analyses will apply. In contrast, transient maneuvers by their very nature are not periodic; hence, many of the basic aerodynamic and acoustic modeling assumptions are invalid. Furthermore, current rotor-noise-prediction codes do not model any flight path that is not rectilinear.

Addressing these issues will involve a two-fold strategy. The first step is to modify the current acoustics model to incorporate the capability to handle curved time-dependent flight paths. The second step is to utilize or develop an appropriate rotor analysis methodology that is suitable for predicting the aerodynamic loads and dynamics of the aircraft during a transient maneuver. In an attempt to keep the scope of this research manageable, it was decided that the primary development for this paper would be the acoustic development and prediction code. Although there are known limitations, readily available aerodynamic tools have been utilized to provide the needed time-dependent rotor-blade loading. The following sections describe both the aeromechanics and acoustic issues that were considered.

\section{AEROMECHANICS ISSUES}

The acoustic prediction requires the determination of the blade motion and loading. A comprehensive analysis code is ultimately required to provide the rotor trim conditions even if relatively sophisticated computational aerodynamic and structural dynamic codes are also used. If the comprehensive analysis code is used alone, then the usual questions of adequacy of the dynamic stall, unsteady aerodynamics, and wake models are compounded by the complication of maneuvering night. For this work, the CAMRAD $2^{4}$ comprehensive analysis code has been utilized. For steady maneuver conditions, the aerodynamic, dynamics, and rotor trim models are appropriate to provide the needed information.

\section{Trimmed Flight}

In typical trim computations, an algorithm of the following form is solved:

$$
R\left(\delta, \lambda_{\mathrm{I}}\left(\beta\left(l\left(\beta, \lambda_{\mathrm{i}}(l(\Pi))\right)\right)\right)\right)=\left(R-R_{\mathrm{trin}}\right),
$$

where $\delta$ represents the aircraft controls, $\lambda_{X}$, the wake geometry, $\beta$, the blade motion, $l$, the aerodynamic load, $\lambda_{2}$, the rotor-wake circulation, $\Pi$, the rotor period, $R_{\text {trim }}$, the desired trim state and $R$ is the system response. The $\beta, \lambda_{X}, \lambda_{i}$, and $l$ functions represent complicated non-linear algorithms which are usually solved using highly damped versions of Newton's method (other methods are also available). The algorithms are "nested" within each other in a way to minimize run-time. Note also the interaction of various functions at several different "levels" of the algorithm. The control vector $\delta$ is systematically updated to achieve solution convergence $\left(R-R_{\text {trim }}\right) \rightarrow 0$. Various trim procedures and levels of complexity have been utilized to solve equation (1).

\section{Transient Maneuver Flight}

One issue that is unique to maneuvering flight is the question of whether the numerical procedure used for normal flight conditions is robust enough for a maneuver. For a vehicle in a transient maneuver, the trim algorithm in equation (1) may be modified to become:

$$
R^{\prime \prime+1}(\delta(t), \beta(t), \lambda(t), l(t))=R^{\prime \prime}+\frac{\partial R^{\prime \prime}}{\partial \delta} \Delta \delta(t) .
$$

Here the solution to the system response is viewed as an initial value problem solved in the time domain. This shift to the time domain makes the additional complexity of the maneuvering flight manageable. Equation (2) is integrated to obtain the response at each time step due to specified control input. A solution set from equation ( 1 ) is used to initiate the problem. For the present application, there are three things to note about this approach. First, the solution is no longer periodic. therefore, each blade must be treated as a separate dynamic component. Second, the solution requires that the control settings are known a priori. while, generally only the required flight path is known. Third, the procedure does not routinely include a subiteration mechanism to stabilize the numerical integration-a shortcoming that leads to the 
accumulation of round-off and convergence errors that will eventually destroy the solution. Past application of this method have been primarily focused on system dynamics stability analyses. The transients used in stability problems are chosen to be short in duration $(\sim 2$ sec) and hence avoid an accumulation of timeintegration error. Short duration events are also of significant interest in acoustics because they potentially have quite high noise levels. For the present effort, only a short-duration transient event is considered.

\section{Model Assumptions}

Typically, the trim and maneuver algorithms described by equations (1) and (2) rely heavily upon semi-empirical models of the various physical phenomena that must be addressed in the solution. A full discussion of all these models is far beyond the scope of this paper but is presented by Johnson ${ }^{4}$. The key modeling assumptions employed in this work will now be reviewed. A notional four-bladed, articulatedrotor aircraft of approximately 14,000 pounds gross weight was used in this study. Since this is an exploratory effort, details of the rotor and aircraft models were not chosen to correspond with any existing aircraft. The model includes main and tail rotors together with fuselage aerodynamics. Dynamic and structural responses for both rotors are computed. A rigid fuselage is assumed. The blade was modeled with 25 unequal radial segments.

For the periodic-trim solutions, 36 azimuthal steps (10-degree resolution) were used for one rotor revolution. The aerodynamic solution was then refined to a 5-degree resolution in a post-trim procedure. Blade loading computed at 5-degree azimuthal increments is insufficient to adequately characterize blade-vortex interactions (BVI), but due to the focus in this paper on the acoustic code development, low azimuthal loading resolution was deemed acceptable at this stage. A full force-free wake geometry model was employed for the main-rotor inflow computations while a uniform inflow model was used for the tail rotor. Lift and drag forces were resolved into the hlade-fixed system and extracted as a function of azimuth angle from the solution using a post-processing plotting code provided with the CAMRAD 2 system. Two harmonics of blade flap. lag, and pitching motion were extracted.

The transient mancuver calculation in CAMRAD 2 was performed with an integration time step of 0.002885 seconds ( -5 -degree arimuth step) and the blade response time history was saved at 0.005770 second intervals ( $\sim$ 10-degree azimuth step). Since the transient maneuver computation was fully timedependent (not quasisteady), both the main and tail rotor aerodynamics were computed using dynamic inflow rather than a more computationally intensive free wake. Lift and drag forces were resolved in a blade fixed system and were extracted for each blade as a function of time. Blade flap, lag, and pitch hinge displacements were also extracted as functions of time for each individual blade. The time history of the aircraft pitch. roll, and yaw orientations and the location of the aircraft center of gravity are computed and saved for the acoustic computation.

\section{ACOUSTIC ISSUES}

Present-day rotor-noise predictions 3.5 are based primarily on the governing equation derived by Ffowes Williams and Hawkings". In principle. the Ffowes Williams-Hawkings (FW-H) equation is appropriate for the noise prediction of any hody in arbitrary motion. however, current rotor-noise-prediction codes have utilized simplifying assumptions in the implementation of the $F W-H$ equation. For example. in the WOPWOP ${ }^{3}$ code, only a single rotor undergoing simple rectilinear motion in the $X-Z$ plane is modeled. Furthermore, the assumption was made during the development of WOPWOP that the rotor motion is periodic and each of the blade motions can be described as a truncated harmonic series. In addition. each of the blades is assumed to experience an identical loading history. These idealizations have been useful in predicting rotor noise, particularly for wind-tunnel models, hut are not particularly appropriate for predicting the noise of maneuvering rotorcraft. In the following subsections. some of the unique challenges of maneuver noise prediction will be presented along with the significant modifications that have been made to the blade-motion modeling in WOPWOP.

\section{Accelerating Flight Capability}

Maneuvering flight is uniquely characterized by aircraft acceleration due to change in direction. change in flight speed, or both. It is important to notice that the aircraft acceleration is the new aspect of the maneuver problem-rotor blade motions and aceclerations must be combined with the additional aircraft motion and acceleration. Aircraft motion and acceleration have been implemented in the version of WOPWOP presented in this paper. The remainder of this subsection describes the implementation of the rotor and aircraft motion in this new version of WOPWOP.

The acoustic formulation ${ }^{7.8}$ in WOPWOP requires $^{3}$ the position. velocity, and acceleration of each point on the rotor blade to be specitied in a frame of reference fixed to the stationary fluid medium (the observer reference frame). A series of time-dependent transformation matrices are used in WOPWOP to relate the time-dependent position of a point in one frame of reference to another reference frame. For example: if $\boldsymbol{\eta}$ is a position vector in reference frame $A$, and $\boldsymbol{x}$ is the position vector of the same point in the coordinates of frame B, then the transformation matrix $T_{B A 4}$ relates $\boldsymbol{\eta}$ to $x$ through the equation $x=T_{B .4} \boldsymbol{\eta}$. The 


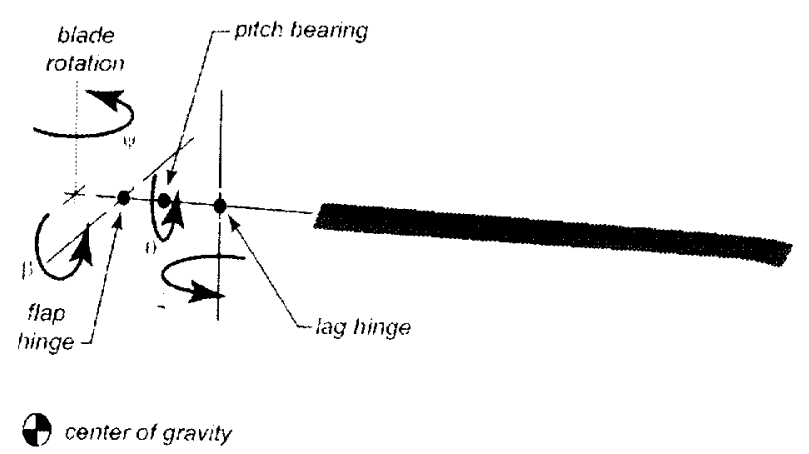

Figure 1. Representative rotor hinge locations.

transformation matrix $T_{B A A}$ includes both the translation and rotation that relate frame $A$ to frame $B$. If there are several frames of reference, say $A, B$, and $C$ then the individual transformation matrices can be concatenated together using matrix multiplication (i.e., $\left.\boldsymbol{x}^{\prime}=T_{C B} T_{B A} \boldsymbol{\eta}\right)$. The velocity and acceleration of a point in frame $B$ are easily computed by taking the time derivative of the position vector, using the chain rule. Hence, the velocity of a point in reference frame $A$ is

$$
\boldsymbol{v}_{B}=\frac{d T_{B A}}{d t} \boldsymbol{\eta}+T_{B A} \frac{d \boldsymbol{\eta}}{d t}
$$

when written in terms of reference frame $B$ coordinates. Here $d \boldsymbol{\eta} / d t$ is the velocity of the point $\boldsymbol{\eta}$ in frame $\mathrm{A}$.

The acceleration of the point $\boldsymbol{\eta}$ can be written

$$
\boldsymbol{a}_{\boldsymbol{B}}=\frac{d^{2} T_{B A}}{d t^{2}} \boldsymbol{\eta}+2 \frac{d T_{B A}}{d t} \frac{d \boldsymbol{\eta}}{d t}+T_{B A} \frac{d^{2} \boldsymbol{\eta}}{d t^{2}}
$$

where $d^{2} \boldsymbol{\eta} / d t^{2}$ is the acceleration of $\boldsymbol{\eta}$ in frame $\mathrm{A}$. Within this framework, it is straightforward to add the additional aircraft motions necessary to model maneuvering flight.

In the original version of WOPWOP (Ref. 3), a position vector in the rotor blade axes system is transformed to the observer frame through blade pitch. lag, flap, and rotation transformations (in that order). The pitch bearing and lag hinge were assumed to be at the same user specified location. The flap hinge was located at the axis of rotation. These idealizations have been removed in the present version of WOPWOP. Now the flap and lag hinges, along with the pitch bearing can be located at the correct physical locations as is schematically represented in figure 1 for the rotor used in the computations presented later. The aircraft body axes, which have their origin at the aircraft center of gravity, are used as an intermediate reference frame for the rotor position and motion vectors.

The aircraft attitude is expressed as the timedependent position of the aircraft body-axes system in an observer reference frame (fixed to the fluid) through aircraft pitch, roll, yaw and velocity (see figure 2 ). The

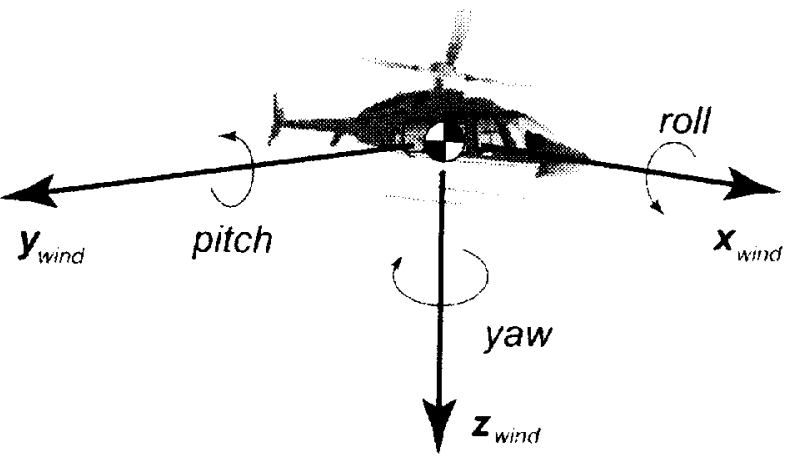

Figure 2. Definition of pitch, roll, and yaw angles that relate the body axes to the wind axes.

$x$ axis of the wind axes system is always aligned with the aircraft velocity vector. The full motion of a point on the source surface (i.e., the rotor blade) in the observer reference frame is found utilizing all of the reference frames from the blade to aircraft body axes to the observer frame. All of the parameters for these transformations may be input into WOPWOP as a time history - no periodicity is implied. Furthermore, the motion and loading for each rotor blade can be input separately. This new functionality enables WOPWOP to compute arbitrary transient maneuvers.

\section{Long Time History Modifications}

Another aspect of maneuvering flight relevant to noise prediction is that the time scale of a maneuver can be much greater than a rotor-blade-passage period. If the same code is used for several hundred rotor revolutions that was designed for only a few blade passages, numerical round-off errors can accumulate and destroy the solution. WOPWOP was modified to minimize round-off error in critical sections of the code to accommodate long-time runs. Computations with over 60,000 points in the acoustic-pressure time history have been run.

An unexpected consequence of long-lime computations is that the required input data stream can become significantly larger. This is especially true now that the blade loading and motion information are represented by an arbitrary time history for each blade and for the aircraft motion. If the blade motion and loading are periodic during the maneuver, then it is possible to utilize the periodicity to minimize the amount of input data. Otherwise, special care is needed to ensure that data input/output is handled efficiently.

\section{Computational Efficiency Enhancements}

Computational efficiency is more important for a maneuver noise prediction for two reasons: 1) the length of the time history that needs to be computed can be significantly longer; and 2) there are nearly double the number of coordinate transformations for the maneuver cases as for the steady-flight case. Two 
major modifications were made to WOPWOP to improve the computational efficiency and thus make the code more suitable for maneuver computations. These modifications are described in the following subsections.

\section{Compact Chordwise Loading Formulation}

Comprehensive analysis codes often do not provide loading as a pressure distribution over a blade surface as the acoustic formulation assumes, but rather as a radial distribution of section lift for the rotor blade. One way to perform rotor noise predictions without the pressure distribution is to assume some sort of representative chordwise pressure variation based upon the section lift. If the chordwise variation of pressure along the blade chord is small relative to the wavelength of the radiated sound, the chordwise distribution can be considered compact. Brentner et al." modeled a compact-chordwise pressure distribution by using a small triangular distribution of pressure located with the peak at the quarter chord of the blade section. Brentner et al." found that this compact chordwise model gave noise predictions accurate within a few decibels (or better), especially below the rotor. The chordwise-compact approximation should be better for low frequencies.

If a chordwise-compact loading model is to be utilized. then some computational efficiency can be gained by changing the acoustic formulation such that numerical integration in the chordwisc direction is not required. The compact-chordwise version of loading noise formulation (formulation $1 \mathrm{~A}$ of Farassat ${ }^{3.8}$ ) is written

$$
\begin{aligned}
4 \pi p_{L}^{\prime}(x, t)= & \frac{1}{r} \int_{f=0}\left[\left.\frac{\dot{L}_{r}}{r\left|1-M_{r}\right|^{2}}\right|_{\mathrm{r}+t} d R\right. \\
& +\int_{f=0}\left[\frac{L_{r}-L_{i}}{r^{2}\left|1-M_{r}\right|^{2}}\right]_{r+t} d R \\
& +\frac{1}{r} \int_{f=0}\left[\frac{L_{r}\left(r \dot{M}_{r}+r\left(M_{r}-M^{2}\right)\right)}{r^{2}\left|1-M_{r}\right|^{3}}\right]_{r+t} d R
\end{aligned}
$$

where $L$ is the section lift vector, $r$ is the distance between the source point and the observer, $M$ is the Mach number vector (source velocity nondimensionalized by the speed of sound $c$ in the quiescent medium) and $R$ is the spanwise integration variable. The subscripts $r$ and $M$ represent dot products of the vector with the unit radiation vector or Mach number vector, respectively. Equation (5) has been implemented as an option in the version of WOPWOP utilized in this paper. Thickness noise still requires chordwise integration, but the number of chordwise integration points needed to resolve the airfoil geometry is less than that to resolve the section loading. The loading noise integration is only carried out along the span of the span of the blade. This not only reduces the number of operations necessary 10 perform the numerical integration of the acoustic formulation, but it also eliminates the need 10 interpolate surface loading data in the chordwise direction.

\section{Self-Scheduling Parallel Implementation}

The WOPWOP code has also been modified for this work in order to utilize parallel computers or clusters of workstations. A self-scheduling approach presented by Long and Brentner ${ }^{10}$ was modified for this version of WOPWOP. The self-scheduling approach utilizes the Single Program Multiple Data (SPMD) parallel programming paradigm in which every processor on the parallel computer (or workstation cluster) will run the same computer program. Through branching in the source code, each processor may execute different portions of the code or use different input data. References 10,11 and 12 describe the details of this parallel programming paradigm in more detail.

In WOPWOP a master-slave algorithm is utilized in order to minimize the necessary source code changes. At the start of the program execution. each of the slave processors first sends a message to the master notifying the master that they are ready for work. The slave processors then wait to get a message from the master. The master reads the case information and sends the waiting slave a job, which is essentially a single WOPWOP run. The slave process runs the noise prediction code-which is now just a subroutine--and writes the results to a file. When all the cases are done the master process stops all of the slave processes. Since there is very little communications-none between slave processors-the parallel efficiency is essentially $100 \%$. In other words, if there are $\mathrm{N}$ processors available, $\mathrm{N}$ cases can be run in the time of one.

This version of WOPWOP includes the capability of reading an array of observer locations and computing the results in parallel. In this case, the noise prediction for each observer location is sent to a separate processor. Long run times can also take advantage of the parallel implementation by breaking a long-time case in to several shorter time segments. The predicted time history for each of the segments can then be pieced back together to form a single time history. The parallel implementation of WOPWOP was utilized for computations of directivity presented later in the paper. Twenty-four processors were available on an Origin 2000 computer, hence contour plots were computed in $1 / 24$ th the time of a single processor.

\section{EXAMPLE COMPUTATIONS}

Several different flight conditions were simulated 10 demonstrate the mancuver modifications to WOPWOP 


\begin{tabular}{ccccccccc}
\hline Case & Condition & $\begin{array}{c}\text { Speed } \\
(\text { knots })\end{array}$ & $\begin{array}{c}\text { Turn Rate } \\
(\mathrm{deg} / \mathrm{sec})\end{array}$ & $\begin{array}{c}\text { Descent Angle } \\
(\mathrm{deg})\end{array}$ & $\begin{array}{c}\text { Pitch } \\
(\mathrm{deg})\end{array}$ & $\begin{array}{c}\text { Roll } \\
(\mathrm{deg})\end{array}$ & $\begin{array}{c}\text { Yaw } \\
(\mathrm{deg})\end{array}$ & $\begin{array}{c}\text { Duration } \\
(\mathrm{sec})\end{array}$ \\
\hline Level Flight & Periodic & 120 & 0 & 0 & -0.61 & -2.68 & 0 & 3 \\
Right Turn & Periodic & 120 & 10 & 0 & 2.21 & 45.36 & 0 & 36 \\
Left Turn & Periodic & 120 & -10 & 0 & 2.24 & -52.06 & 0 & 36 \\
Descent & Periodic & 80 & 0 & -3 & -0.81 & 0. & 6.51 & 2 \\
$\begin{array}{c}\text { Arrested } \\
\text { Descent }\end{array}$ & $\begin{array}{c}\text { Time Domain } \\
\text { Transient }\end{array}$ & $\mathbf{8 0}$ & 0 & -3 & see & see & see & 2 \\
\hline
\end{tabular}

Table 1. Flight conditions and aircraft attitude.

\begin{tabular}{cccccccc}
\hline $\begin{array}{c}\text { Flight } \\
\text { Condition }\end{array}$ & $\begin{array}{c}\text { Speed } \\
\text { (knots) }\end{array}$ & $\begin{array}{c}\text { Collective } \\
(\mathrm{deg})\end{array}$ & $\begin{array}{c}\text { Lateral } \\
(\mathrm{deg})\end{array}$ & $\begin{array}{c}\text { Longitudinal } \\
(\mathrm{deg})\end{array}$ & $\begin{array}{c}\text { Pedal } \\
\text { (deg) }\end{array}$ & $\begin{array}{c}\text { Thrust } \\
\text { (lbs) }\end{array}$ & $\begin{array}{c}\text { Power } \\
\text { (hp) }\end{array}$ \\
\hline Level Flight & 120 & 9.36 & -1.95 & 6.1 & -2.5 & 14503 & 13.30 \\
Right Turn & 120 & 14.03 & -3.31 & 10.23 & -8.41 & 21060 & 29.58 \\
Left Turn & 120 & 14.59 & -3.89 & 11.3 & -9.99 & 21420 & 3471 \\
Descent & 80 & 6.51 & -2.24 & 2.27 & -2.83 & 14673 & 779 \\
\hline
\end{tabular}

Table 2. Rotor parameters for steady conditions.

and investigate the challenges of maneuver noise prediction. A list of these conditions along with relevant aircraft state information is listed in Table 1. These example computations are intended to demonstrate the significant impact a flight maneuver can have on the radiated noise from a rotorcraft and to exercise the WOPWOP and CAMRAD 2 codes in realistic maneuver situations. The computations should be considered preliminary.

In the remainder of this section, the various flight conditions will be evaluated. Level flight will be compared to right and left coordinated turns and a descent condition typical of approach. A transient maneuver is also demonstrated.

\section{Level and Turning Flight Comparison}

The level-flight case represents the baseline condition for the study. The aircraft is in trimmed-level flight at a moderate speed of 120 knots. The right and left turn cases represent variations from the level-flight condition. which reflect the effect of a true maneuver. although the rotor motion and loading are periodic. The main rotor rotates in the counterclockwise direction, as viewed from above the rotor. The turn radius and turn rate were $1160.5 \mathrm{ft}$ and $10 \mathrm{deg} / \mathrm{sec}$, respectively, for both the right and left lurns. The rotor control settings and the required power for the steady cases are shown in Table 2.

The normal force and chordwise force computed by CAMRAD 2 are shown for each flight condition in figures 3 and 4 . The most dramatic difference between the turn conditions and level flight is the significantly increased loading on the rotor blade. This is expected since the rotor must provide the thrust to offset both the aircraft weight and centrifugal acceleration. A careful comparison of the right and left turn reveals that the loading for the left turn is slightly higher and the peak loading is shift toward the centerline of the vehicle. Both the normal force and chordwise force are utilized in WOPWOP for the noise prediction.

Acoustic predictions were computed on a $600-\mathrm{ft}$ wide by 900 -ft long plane $100 \mathrm{ft}$ below the helicopter. Acoustic pressure time histories were computed at 3015 observer locations on the measurement plane. Computations were made using both moving observers (i.e., the observer locations maintain the same spatial relationship with the helicopter) and stationary observers (flyover case). When the observer locations are in motion, the acoustic signal is periodic (i.e., with fixed blade passage periodic). Hence, for steady flight conditions in this paper, the overall sound pressure level (OASPL) was computed for a moving observer at each point on the measurement grid. One bladepassage period was utilized in the computation of OASPL. Only the main-rotor noise was predicted.

Figure 5 shows a comparison of the OASPL contours for level and turning flight. The small circle in the center of the contour plot represents the projection of the main rotor disk onto the measurement plane. It is interesting that the turning flight peak OASPL levels are as much as 10 decibels (dB) higher than the level flight case. This is a result of the significant increase in rotor loading for turning flight. Although loading is nearly the same for left and right turns, there is a significant difference in the OASPL directivity of the right and left turns. More work is required to explain this difference. 

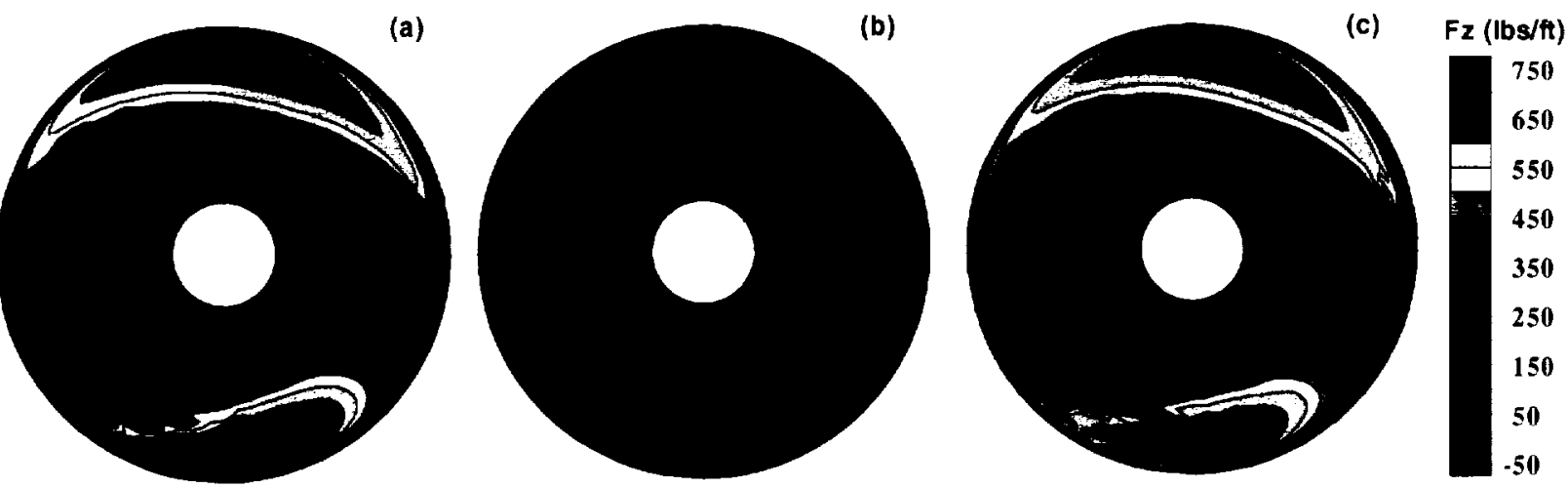

Figure 3. Comparison of normal force per unit span for level and turning flight (direction of flight from bottom to top; zero degrees azimuth is at the bottom). (a) left turn; (b) level flight; (c) right turn
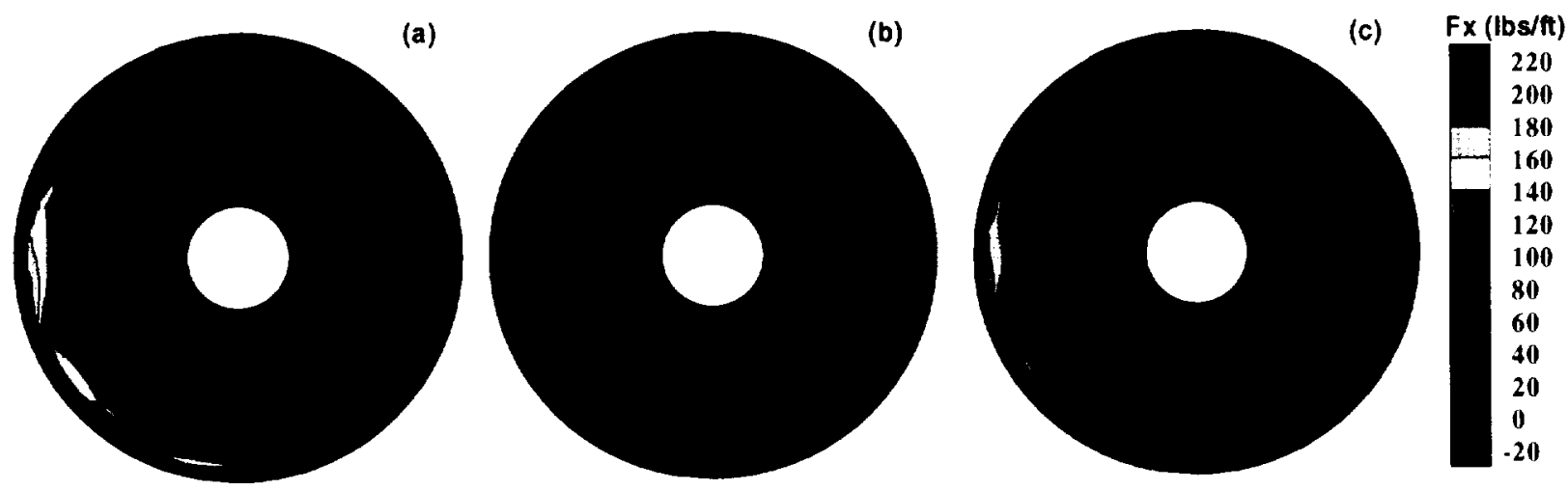

Figure 4. Comparison of chordwise force per unit span for level and turning filght (direction of flight from bottom to top; zero degrees azimuth is at the bottom). (a) left turn; (b) level night; (c) right turn.

Overall sound pressure level tends to emphasize low-frequency rotor noise. The A-weighted spectrum has also been used to give an A-weighted overall sound pressure level. The A weighting emphasizes the frequencies to which the human ear is most sensitive. Hence, the A-weight OASPL emphasizes higher frequencies of rotor noise. Impulsive noise, such as BVI noise and HSI noise, will have more influence on Aweighted noise sound pressure levels. A-weighted OASPL contours for the level and turning flight cases are shown in figure 6 . Again, the turning flight cases are significantly higher than the level flight case. The peak right turn noise is also 4 to $6 \mathrm{~dB}$ higher than that of the left turn. Since the main rotor rotates counterclockwise, the rotor would be expected to encounter more advancing-side BVI and less-retreating side BVI for the right turn. The converse would be true for the left turn. It is difficult to tell from figure 3 if this is reflected in the loading. Although the aerodynamic computations for this paper do not have the spatial or temporal resolution needed for the accurate prediction of BVI noise, it is possible that the high frequency content reflected in the A-weighted
OASPL contours is a numerical artifact of the unresolved BVI.

\section{Level and Descending Flight Comparison}

A 3-degree descent at a forward speed of 85 knots was investigated as a case representative of an approach. The descent case represents a condition of high rotor-wake interaction, which should lead to high BVI noise conditions. The level flight and 3-degree descent section normal forces are compared in figure 7 . Although the forward speed is significantly higher for the level flight case, it is still interesting to compare these two conditions from an acoustic point of view. Notice in figure 7 that the peak normal force is approximately the same, although the distribution of loading over the rotor disk is quite different. In particular, on the advancing side of the rotor (right side) at approximately 70-90 degrees azimuth and on the retreating side of the rotor in the fourth quadrant (lower left corner) there is a convergence of contour lines indicating rapid changes in value. These areas are where BVI are expected to occur, therefore, it seems CAMRAD 2 is giving some indication of the BVI loading (although it is clearly not well resolved). 
(a)

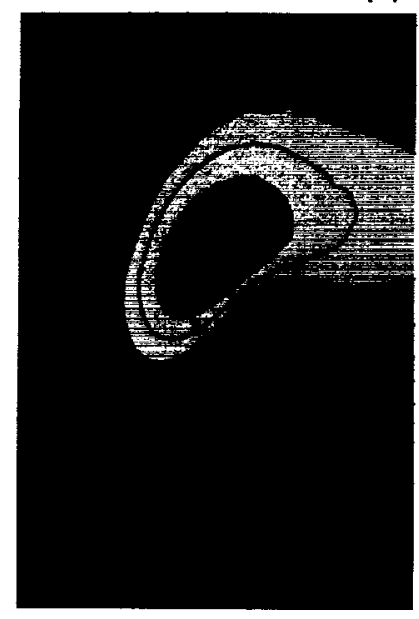

(b)

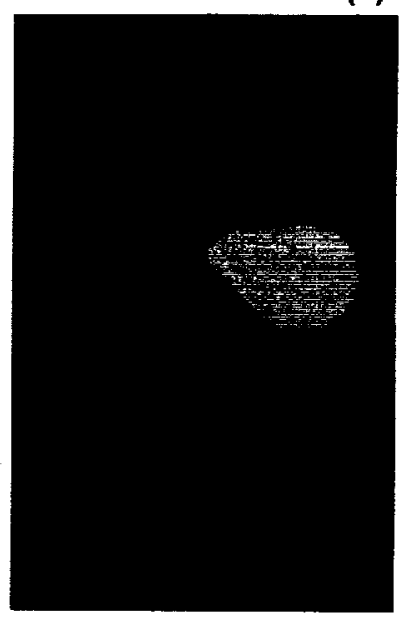

(c)

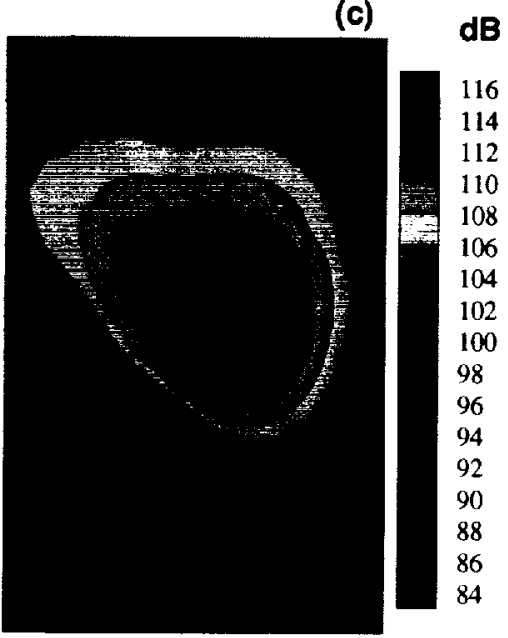

Figure 5. Overall sound pressure level contours. (a) left turn; (b) level flight; (c) right turn.

(a)

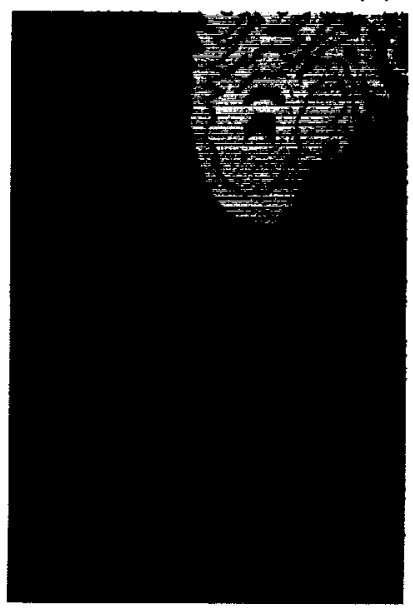

(b)

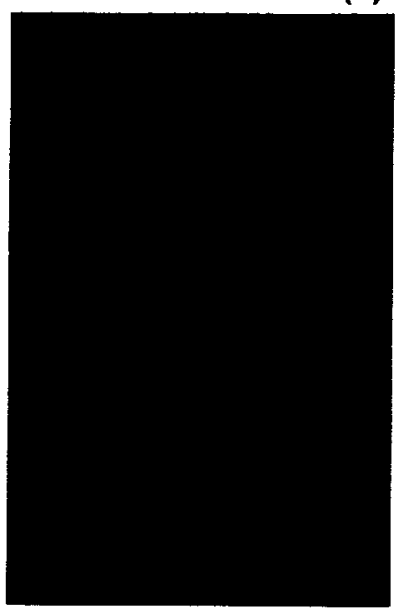

(c)

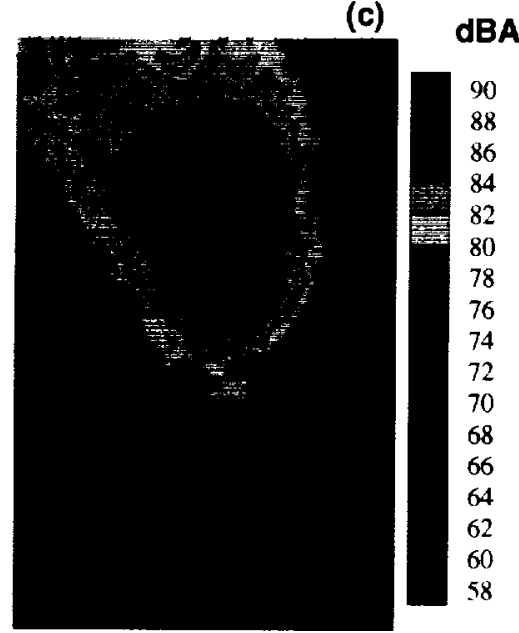

Figure 6. A-weighted overall sound pressure level contours. (a) left turn; (b) level flight; (c) right turn.

Figures 8 and 9 show the comparison of the OASPL and the A-weighted OASPL, respectively. Although the forward speed is 35 knots higher for the level flight case, the peak OASPL level is only $2 \mathrm{~dB}$ higher than that for the 3-degree descent. The directivities for the two cases have some similarities. In figure 9, the Aweighted directivity has a significant area where the peak level is 4 to $5 \mathrm{~dB}$ higher for the descent case than for the level flight case. The descent case clearly has higher frequency content due to BVI-like loading.

\section{Transient Maneuver}

The final maneuver studied in this paper is a 3degree arrested descent. For this maneuver, a collective pulse control input was used to introduce the transient and to arrest the descent of the helicopter. The collective pulse is shown in figure 10 . This maneuver was chosen to be a simple, short-time transient maneuver and is representative of changing from a higher descent rate to a lower descent rate during a segmented approach. The total time for the maneuver was 2 seconds, with the collective pulse started 0.5 seconds into maneuver and lasting for 1 second. Figure 11 shows the change in aircraft altitude while figure 12 shows the aircraft attitude during the maneuver. The arrested descent condition is the only true transient studied in the present paper. In an attempt to reduce the complexity of the comprehensive analysis computation, a dynamic inflow model was used rather than a free wake for the transient maneuver.

The unique aspect of the transient maneuver-nonperiodic blade motion and loading-can be seen in a comparison of the flap angle for each blade and the section normal force for each blade in figures 13 and 14, respectively. The flap angle and normal force have 


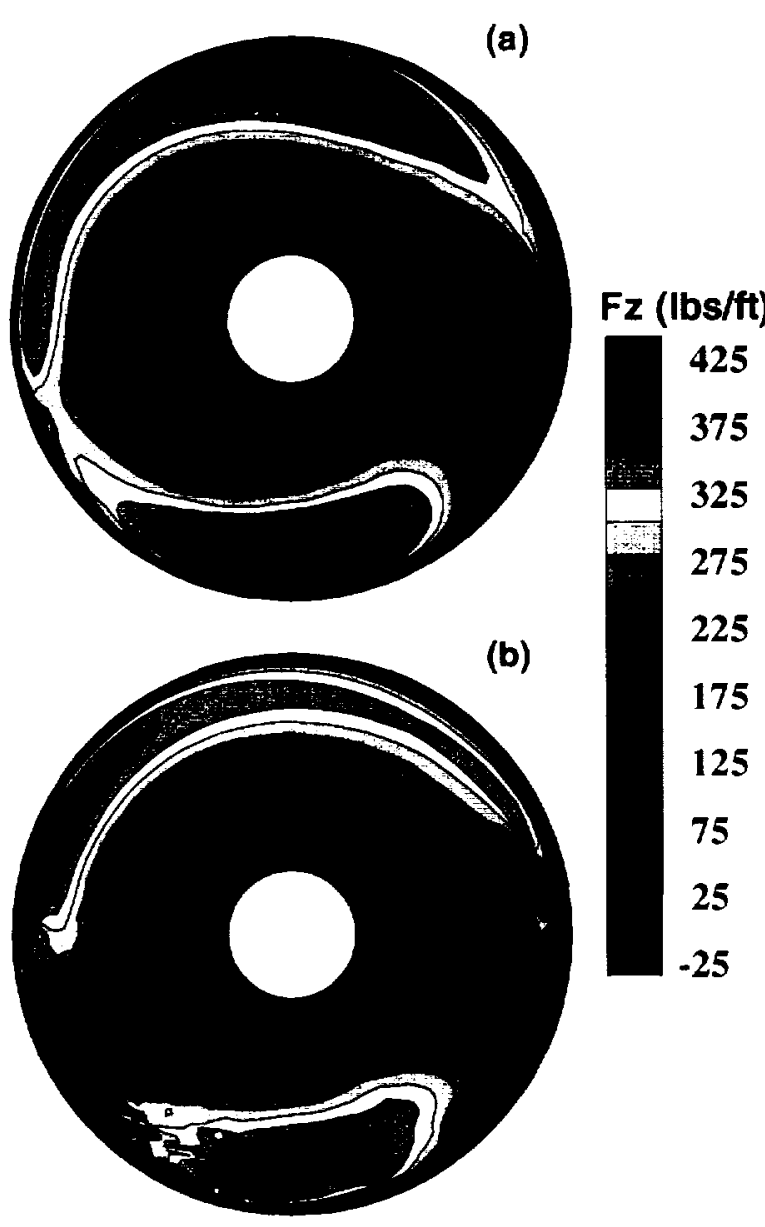

Figure 7. Comparison of normal force per unit span for level and descending flight (direction of flight from bottom to top; zero degrees aximuth is at the bottom). (a) level flight; (b) 3-degree descent.

been plotted against blade azimuth angle to emphasize the blade-to-blade differences at a particular azimuth angle. The blade motion and loading for each blade are identical, both before and after the transient control input. During the transient control, however, each blade has a unique response. It is interesting to note that the response of each blade is nearly the same during the middle of the transient (azimuth angles from 1800 to 2160 degrees). This happens because the collective pulse has a very small control change during the middle of the event.

To demonstrate the effect the transient collective control has on the noise, the acoustic pressure for both a 3-degree descent and the arrested descent (both airloads computations made with the dynamic inflow model) is shown in figure 15. The observer location in this case is 100 feet below the helicopter center of gravity at time $t=0$. Hence, the acoustic pressure signal is for a time shortly after the helicopter has flown over the observer. For this condition, thickness noise is negligible and (a)

(b)

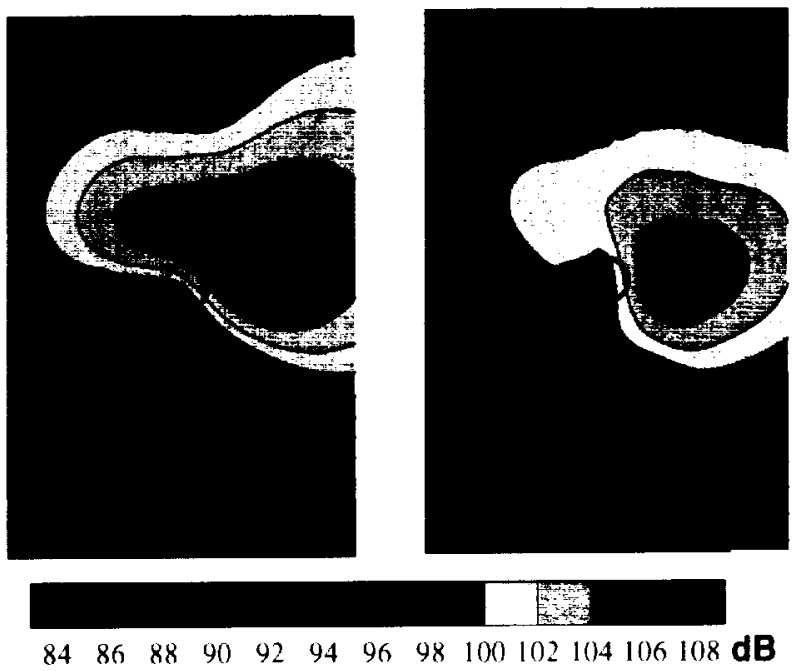

Figure 8. Overall sound pressure level contours. (a) level flight; (b) 3-degree descent.

(a)

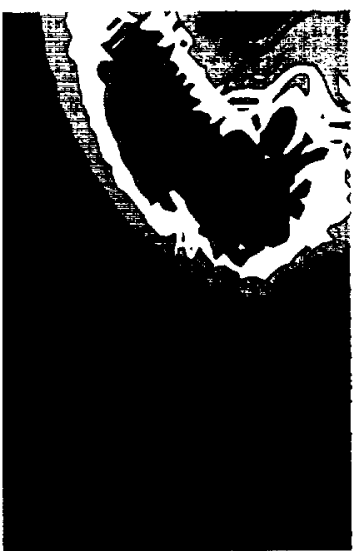

(b)

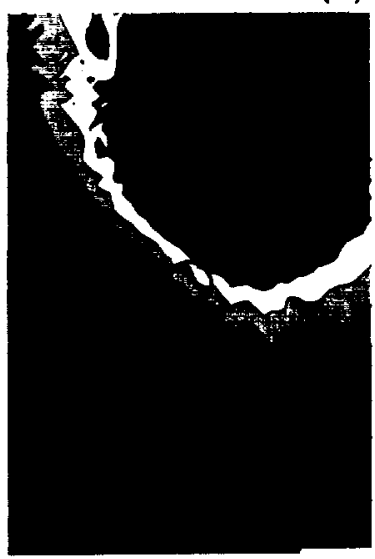

$\begin{array}{llllllllllllllll}54 & 56 & 58 & 60 & 62 & 64 & 66 & 68 & 70 & 72 & 74 & 76 & 78 & 80 & 82 & \text { dBA }\end{array}$

Figure 9. A-weighted sound pressure level contours. (a) level flight; (b) 3-degree descent.

loading noise is dominant. During the time $0.7<t<1.7 \mathrm{sec}$, the amplitude of the acoustic pressure increases to over three times that of the normal 3-degree descent. This demonstrates that a transient maneuver can have a potent impact on the rotor noise output.

\section{CONCLUDING REMARKS}

This paper has outlined the approach necessary to compute the noise from a maneuvering rotorcraft. All of the development work is applicable to both steady and transient maneuvers. The comprehensive analysis code, CAMRAD 2, was utilized without modification 


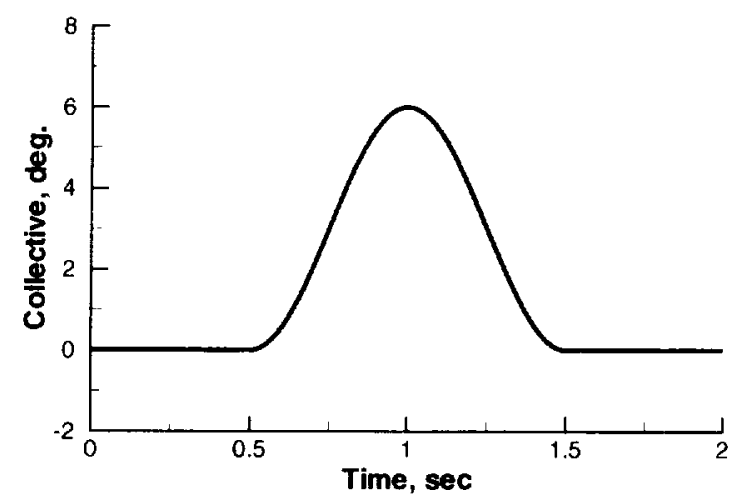

Figure 10. Collective input control for transient maneuver.

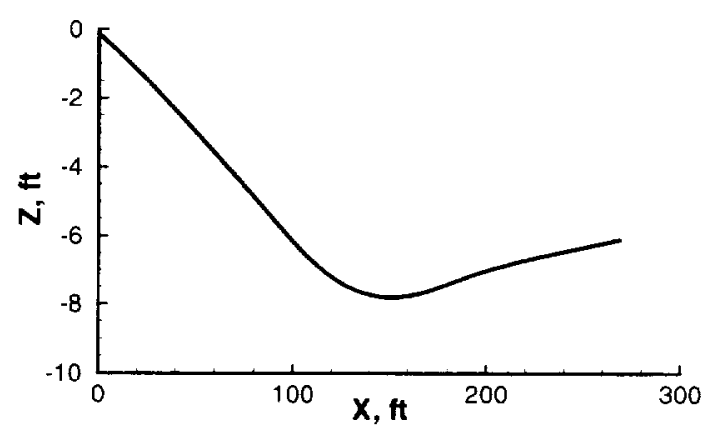

Figure 11. Change in aircraft altitude as the aircraft travels forward.

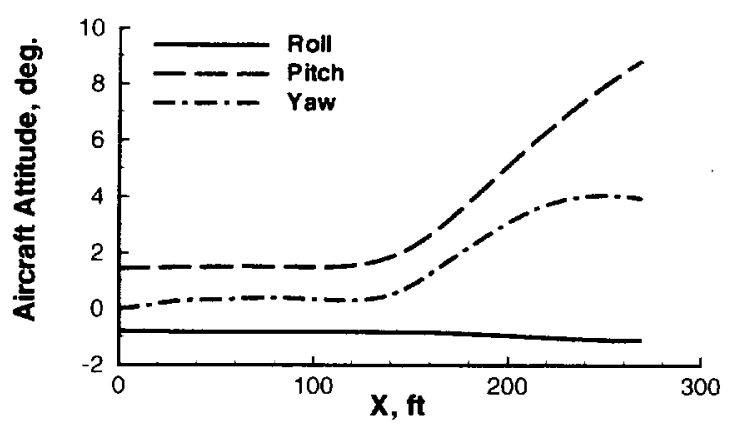

Figure 12. Change in aircraft attitude (roll, pitch, and yaw) as the aircraft travels forward.

to the code for the maneuver case, albeit engineering judgement and some trial and error were needed to successfully predict the rotor airloads in the maneuvering flight conditions. Further work is needed to explore the real limitations of the comprehensive analysis.

The major focus of this effort was the development of an enhanced version of WOPWOP. Full aircraft motion. including arbitrary transient motion, can be modeled. Furthermore, rotor blade motions also can be arbitrarily specified as a time history. Hinge locations can be specified at the actual locations. The only simplifying assumption that remains is that the blade is a rigid body. Additional enhancements to take advantage of parallel computers and clusters of

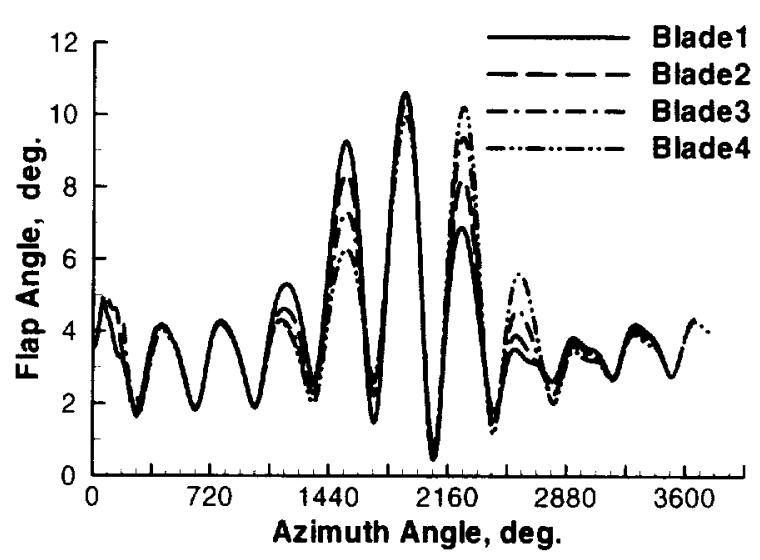

Figure 13. Transient flap angle plotted as a function of blade azimuth position.

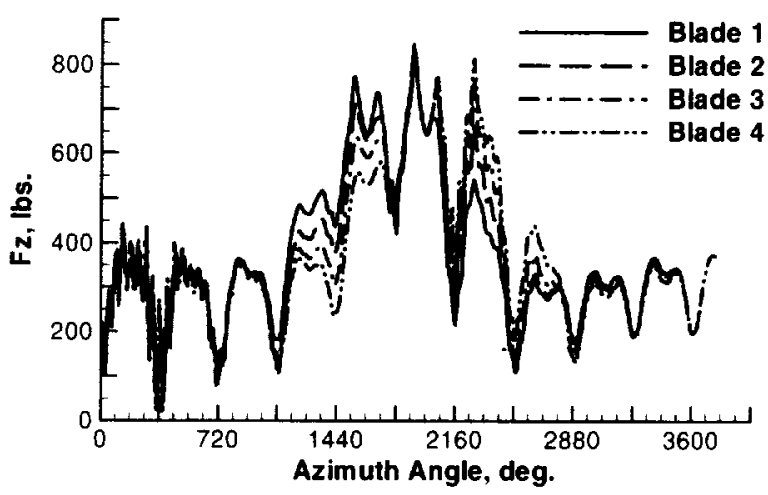

Figure 14. Transient normal force $(r / R=0.9475$ ) plotted as a function of blade azimuth position.

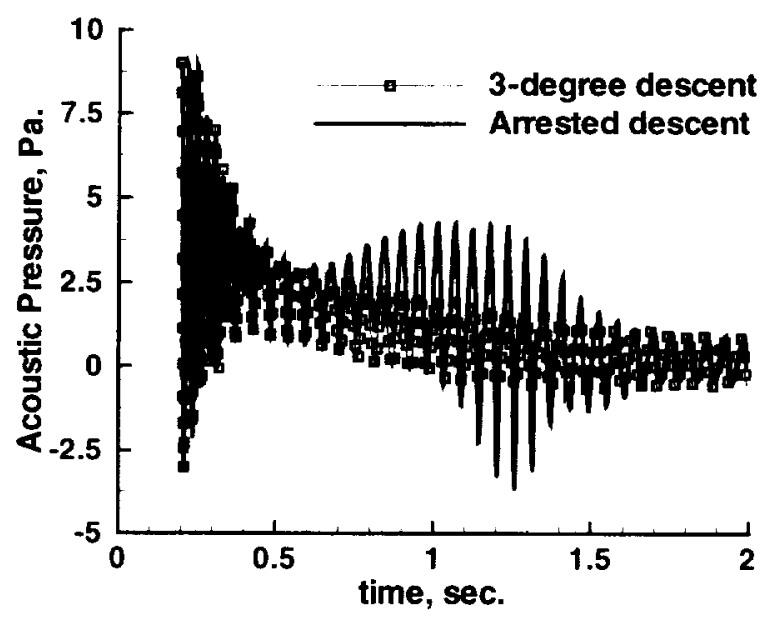

Figure 15. Acoustic pressure comparison for 3degree descent and arrested descent. 
workstations, in addition to a new compact-chordwise loading formulation, have been added to the code.

This paper demonstrated the importance of maneuver by predicting the noise from a notional rotorcraft in turning flight and descent and comparing it to level flight. A substantial increase in the rotor noise for turning flight was found when compared to level flight at the same speed. This is due to the increased loading a rotor must produce in a turn. The noise for a transient maneuver (a 3-degree arrested descent) was computed. A comparison of the acoustic pressure for the 3-degree descent and the arrested descent showed an increase in acoustic pressure amplitude by more than 300 percent. This computation demonstrates the large impact maneuver can have on rotor noise radiation. This paper is intended only as an initial step toward the goal of first-principles noise prediction from maneuvering rotors.

\section{REFERENCES}

${ }^{1}$ Gaurav Gopalan, Fredric H. Schmitz and Ben Wel-C. Sim, "Flight Path Management and Control

Methodology to Reduce Helicopter Blade-Vortex Interaction (BVI) Noise," American Helicopter Society Vertical Lift Design Conference, San Francisco, CA, January 19-21, 2000.

'Ram D. Janakiram and Hamza Khan, "Prediction and Validation of Helicopter Descent Flyover Noise," AHS International 56th Annual Forum Proceedings, Virginia Beach, VA. May 2-4, 2000.

"Kenneth S. Brentner, "Prediction of Helicopter Rotor Discrete Frequency Noise." NASA TM 87721. October, 1986.

${ }^{+}$Wayne Johnson, "Comprehensive Analytical Model of Rotorcraft Aerodynamics and Dynamics," Vol. I VII. Johnson Aeronautics, Sept. 1998.

${ }^{5}$ Kenneth S. Brentner and F. Farassat, "An Analytical Comparison of the Acoustic Analogy and Kirchhoff Formulation for Moving Surfaces," AlAA Journal, Vol. 36. No. 8, pp. 1379-1386, Aug. 1998.

"J. E. Ffowes Williams and D. L. Hawkings, "Sound Generation by Turbulence and Surfaces in Arbitrary Motion," Philosophical Transactions of the Roval Society, London, Series A, Vol. 264, No 1151, pp. 32I342. May 1969.

${ }^{7}$ F. Farassat, "Theory of Noise Generation from Moving Bodies with an Application to Helicopter Rotors," NASA TR R-451, 1975.

${ }^{8}$ F. Farassat and George P.Succi, "The Prediction of Helicopter Discrete Frequency Noise," Vertica, Vol. 7. No. 4, pp. 309-320. 1983.

${ }^{9}$ Kenneth S. Brentner, Casey L. Burley and Michael A. Marcolini, "Sensitivity of Acoustic Prediction to Variation of Input Parameters," Journal of the American Helicopter Society, Vol. 39. No. 3, pp. 4352 , July 1994.
${ }^{10}$ Lyle N. Long and Kenneth S. Brentner, "SelfScheduling Parallel Methods for Multiple Serial Codes with Application to WOPWOP." AIAA Paper 20(X)0346 , Jan. 2000.

"http://www.mhpcc.edu/training/

12 http://www.personal.psu.edu/lnl/424/ 
2 